\title{
Exciton localization in excited-state dynamics of a tetracene trimer: A surface hopping LC-TDDFTB study
}

\author{
Evgenii Titov, Alexander Humeniuk, and Roland Mitrić \\ Institut für Physikalische und Theoretische Chemie, Julius-Maximilians-Universität \\ Würzburg, Emil-Fischer-Strasse 42, 97074 Würzburg, Germany. \\ E-mail: roland.mitric@uni-wuerzburg.de
}

\begin{abstract}
Excitons in molecular aggregates of chromophores are key participants in such important processes as photosynthesis or the functioning of organic photovoltaics devices. Therefore, exploration of exciton dynamics is crucial. Here we report on exciton localization during excited-state dynamics of the recently synthesized tetracene trimer [Liu et al., Org. Lett., 2017, 19, 580]. We employ the surface hopping approach to nonadiabatic molecular dynamics in conjunction with the long-range corrected time-dependent density functional tight binding (LC-TDDFTB) method [Humeniuk and Mitrić, Comput. Phys. Commun., 2017, 221, 174]. Utilizing a set of descriptors based on the transition density matrix, we perform comprehensive analysis of exciton dynamics. The obtained results reveal an ultrafast exciton localization to a single tetracene unit of the trimer during excited-state dynamics, along with exciton transfer between units.
\end{abstract}

\section{Introduction}

Excitons in molecular assemblies play a prominent role, ${ }^{1,2}$ since they are involved in such processes as photosynthesis (excitons formed via light absorption are transferred into chemical energy $)^{3}$ and functioning of organic photovoltaics (excitons are split to provide electrical 
power). ${ }^{4}$ Molecular excitons themselves can be viewed as electronically excited states of a whole molecular aggregate, contrasted to excited states of individual chromophores. Physical models for description of molecular excitons are known since the 1960s due to Davydov ${ }^{5}$ and Kasha. ${ }^{6}$ These models are based on properties of an individual chromophoric unit and some approximate electronic coupling between units. The improved theories, refining the inter-molecular coupling to include for example the charge transfer states, are the subject of nowadays investigations. ${ }^{7}$ For systems containing only a few chromophores the full first-principles treatment (with its own limitations though), either by means of wavefunction-based or DFT methods, can be affordable (see, e.g., refs. 8,9) and may serve to assess the results obtained by approximate exciton models.

The first principles approaches yield information about stationary electronic states of aggregates at a fixed nuclear configuration. To elucidate exciton dynamics one should go beyond this stationary picture and account for nuclear motion and its coupling with the electron dynamics. This can be achieved by performing (nonadiabatic) molecular dynamics simulations, either assuming classical nuclei ${ }^{10,11}$ or treating selected vibrational degrees of freedom quantum-mechanically. ${ }^{12-14}$

Exciton localization/delocalization dynamics has recently been studied for a series of organic molecular systems by several groups, including Tretiak and co-workers, ${ }^{15-28}$ Lan and co-workers, ${ }^{29-31}$ Barbatti and co-workers, ${ }^{32,33}$ as well as our group. ${ }^{34,35}$ In order to analyze the exciton dynamics, Tretiak et al. have performed nonadiabatic surface hopping ${ }^{36}$ molecular dynamics simulations (employing the AM1 semiempirical Hamiltonian ${ }^{37}$ and configuration interaction singles (CIS)) and have used the transition density matrix as a quantity for the characterization of exciton dynamics. ${ }^{38}$ Specifically, the transition density matrices - quantities that reflect the changes in electron density upon excitation and allow one to conclude about spatial localization/delocalization of exciton - are computed along surface hopping trajectories, i.e. for different nuclear configurations which arise during dynamics. In this way, the overall complex picture of coupled dynamics comprising the changes of both molecular geometry and electronic wavefunction is obtained. This approach has proven very useful in tackling peculiarities of exciton localization/delocalization in relatively large molecular systems.

Here, we apply our recently developed LC-TDDFTB nonadiabatic dynamics ${ }^{39-41}$ and introduce some new descriptors of localization/delocalization, 
to elucidate exciton dynamics in a recently synthesized covalentlylinked tetracene trimer. ${ }^{42}$

This molecule, composed of 3 tetracene units, is of interest for photovoltaics, in particular, for singlet fission (see, e.g., refs. 43-45 for the description of the latter) due to its ability to generate triplet states via the intramolecular singlet fission pathway.

In the present paper we want to address the initial phase of the photophysics in this linear tetracene trimer molecule that involves exciton localization/delocalization dynamics induced by the photoexcitation of a bright singlet excited state. The LC-TDDFTB method allows one to treat efficiently rather large molecular systems, including molecular aggregates composed of several chromophores, while not suffering from the charge-transfer problem. ${ }^{46-49}$ As a drawback, analogous to linear response TDDFT, the LC-TDDFTB method does not allow for description of doubly excited states (see, e.g., refs. 50-52), which are important for processes such as singlet fission, and which can be calculated with, e.g., spin-flip configuration interaction approaches ${ }^{53-55}$ or multireference perturbation theory, ${ }^{52,56}$ which generally are much more time-demanding. Thus, by using LC-TDDFTB, we constrain the manifold of excited states to the singly excited singlet states, and focus on the dynamics within this subset. This approximation is expected to yield reasonable results for the excited-state dynamics if the doubly-excited states do not couple strongly with the bright exciton states, which are initially excited. The exciton dynamics is analyzed in terms of the transition density matrix. The analysis sheds light on ultrafast exciton localization with subsequent exciton transfer between fragments of the molecule.

\section{Methods}

\subsection{Quantum chemical and surface hopping cal- culations}

The trimer structure was optimized in the electronic ground state using the $\omega$ B97X-D density functional ${ }^{57}$ and the def2-TZVP basis set. ${ }^{58}$ The optimized geometry is shown in Fig. 1a. 

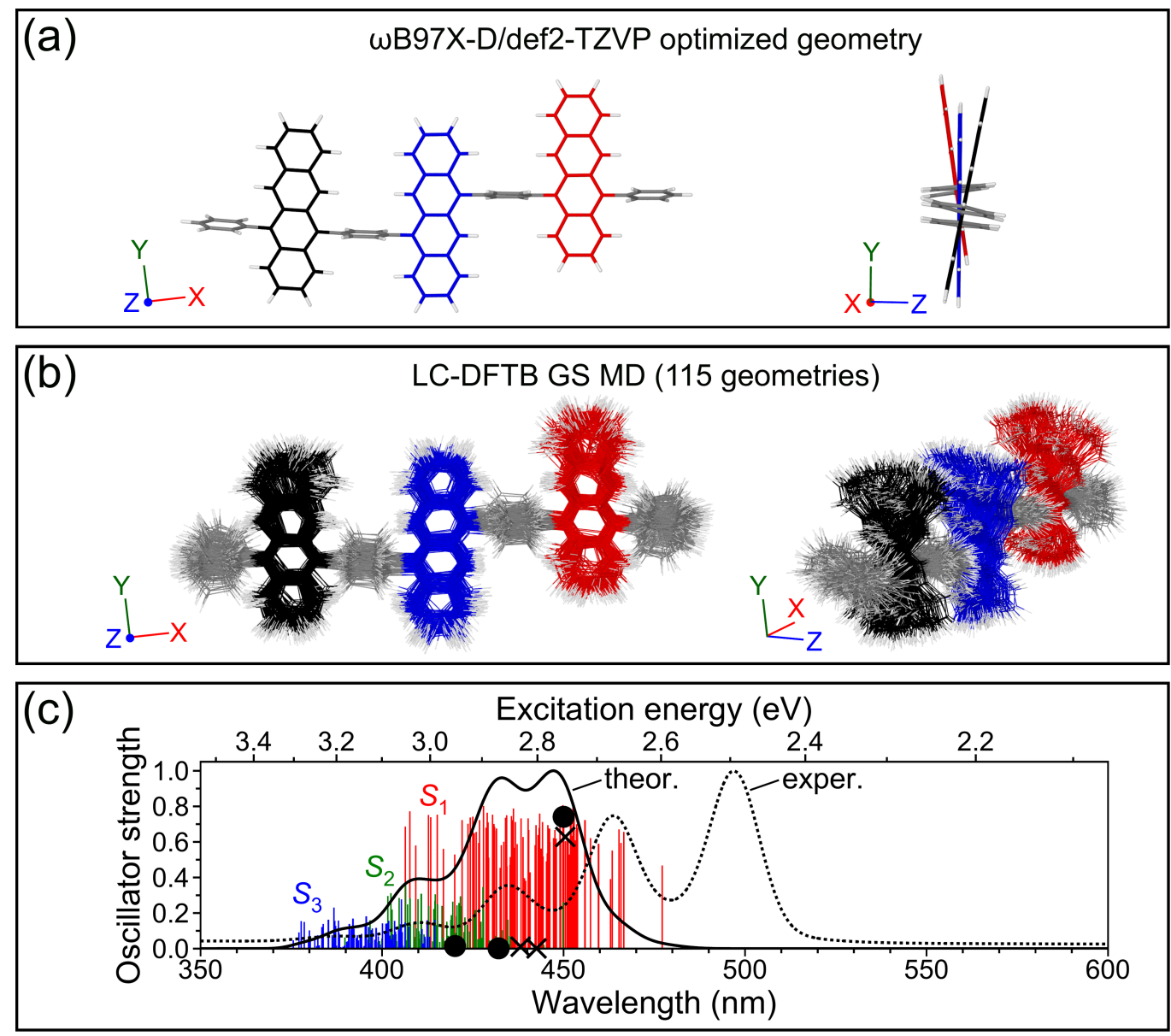

Figure 1: (a) Two different views of the geometry of the tetracene trimer optimized at the $\omega \mathrm{B} 97 \mathrm{X}-\mathrm{D} /$ def2-TZVP level. The tetracene units are colored: left in black, middle in blue, right in red. (b) Ensemble of 115 geometries sampled from a long-range corrected DFTB ground-state molecular dynamics simulation (LC-DFTB GS MD) at constant temperature (300 K). Two different views are shown. (c) Vertical absorption spectra calculated for 115 geometries sampled from LC-DFTB GS MD. The size of active space for LCTDDFTB calculations is $30 \times 30$. The solid curve is the broadened spectrum (for gas phase) calculated as $\sum_{i} f_{i} \exp \left(-\frac{1}{2 \sigma^{2}}\left(\frac{1}{\lambda}-\frac{1}{\lambda_{i}}\right)^{2}\right)$ with $\sigma=250 \mathrm{~cm}^{-1}$ and normalized to have maximum absorbance of 1 (here, $\lambda$ is the wavelength, $\lambda_{i}$ and $f_{i}$ are computed excitation wavelengths and oscillator strengths, respectively, $\sigma$ is a broadening parameter). The dotted curve is the normalized experimental spectrum (recorded in toluene) of Liu and co-workers, taken from Fig. 1c of ref. 42. The black circles represent the first three vertical transitions calculated with LC-TDDFTB $(30 \times 30)$ at the $\omega$ B97X-D/def2TZVP optimized geometry (shown in (a)). The black crosses show the first three vertical transitions calculated with TD- $\omega$ B97X-D/def2-TZVP at the same geometry of (a). 
As a starting point for the optimization we used the B3LYP/6$31 \mathrm{G}^{* *}$ geometry of ref. 42 . The B3LYP,${ }^{59,60}$ CAM-B3LYP $,{ }^{61} \omega \mathrm{B} 97,{ }^{62}$ $\omega \mathrm{B} 97 \mathrm{X}^{62}$ and $\omega \mathrm{B} 97 \mathrm{X}-\mathrm{D}^{57}$ functionals as well as the $\mathrm{ADC}(2)$ method, ${ }^{63,64}$ linear response TDHF (see, e.g., refs. 65-67), and AM1 ${ }^{37} /$ CISDT $(12 \times 12)$ (i.e. 12 highest occupied and 12 lowest unoccupied orbitals were used in configuration interaction singles doubles triples) were used to calculate vertical excitation energies and oscillator strengths (of transitions to low-lying excited states), to compare with LC-TDDFTB results.

In addition, GMC-QDPT2 (generalized multiconfigurational quasidegenerate perturbation theory of second order $)^{68-70}$ calculations have been performed to account for the doubly-excited states. Starting orbitals for the MCSCF calculation were generated by a closed-shell restricted Hartree-Fock calculation with the Dunning/Hay double zeta basis set. ${ }^{71}$ The state-averaged MCSCF calculation included the lowest 9 singlet states using a complete active space consisting of 6 electrons in the 6 frontier orbitals HOMO-2, HOMO-1, HOMO, LUMO, $\mathrm{LUMO}+1, \mathrm{LUMO}+2$. The frontier orbitals are linear combinations of the tetracene HOMO and LUMO orbitals on each monomer unit.

(TD)DFT and TDHF calculations were performed with Gaussian 09, ${ }^{72}$ ADC(2) calculations with TURBOMOLE V7.0, ${ }^{73}$ AM1/CISDT with MNDO, ${ }^{74}$ and LC-TDDFTB calculations with the DFTBaby software. ${ }^{40,41}$ GAMESS $^{75}$ was employed for GMC-QDPT2 calculations.

The dynamics of the trimer was modeled employing the LC-TDDFTB method. ${ }^{39,40}$ First, a ground-state molecular dynamics run was performed at a constant temperature of $300 \mathrm{~K}$ to equilibrate the system and sample initial conditions (nuclear positions and velocities) for subsequent nonadiabatic surface hopping dynamics. The Berendsen thermostat was used to control the temperature. ${ }^{76}$ As initial condition for the ground-state dynamics, the optimized $\omega$ B97X-D/def2-TZVP geometry and randomly generated velocities (corresponding to $300 \mathrm{~K}$ ) were used. The time step for the ground-state molecular dynamics was set to 0.2 fs. Initial conditions (115 in total) for surface hopping dynamics were sampled every 100 fs starting after 5 ps of the ground-state molecular dynamics. The surface hopping is a stochastic approach and ideally one should use as many trajectories as possible. In practice, however, due to substantial computational demand about 100 trajectories are often used in surface hopping studies. ${ }^{9,40,77,78}$ The ensemble of initial geometries is shown in Fig. 1b. We note that the ground-state molecular dynamics allows us to sample geometries with 
different dihedral angles between tetracene units.

For the calculation of excited states we used a reduced active space composed of the highest 30 occupied and the lowest 30 virtual orbitals, denoted as $30 \times 30$ in what follows. This space provides good compromise between accuracy of excitation energies and the computational time needed to calculate excitation energies and its gradients, which are needed to carry out nonadiabatic dynamics simulations. Excitation energies and corresponding oscillator strengths were computed for selected (115 in total) geometries in order to simulate thermally broadened absorption spectrum. For all the sampled nuclear configurations the $S_{0} \rightarrow S_{1}$ transition was found to be the most intense one (see Fig. 1c). Therefore, all excited-state trajectories were launched from the $S_{1}$ state. The nonadiabatic dynamics was simulated employing Tully's surface hopping approach, ${ }^{36}$ with modified calculation of hopping probabilities. ${ }^{79}$ A local diabatization scheme was employed to propagate the electronic wavefunction. ${ }^{80,81}$ The time step for surface hopping dynamics was set to 0.1 fs. Each trajectory was propagated up to 500 fs.

The ground and 3 lowest singlet excited states were included. We selected the first three excited states based on the relatively large separation of higher excited states from the $S_{3}$ state. Higher lying excited states may in fact be predominantly of doubly-excited character, as also confirmed by multiconfigurational perturbation theory calculations (see section 3).

\subsection{Transition density matrix}

The central quantity for the analysis of the exciton dynamics performed here is the reduced first-order spinless transition density matrix between ground and excited electronic states, defined as (see, e.g., 82).

$$
\begin{aligned}
\rho^{0 I}\left(\vec{r}, \vec{r}^{\prime}\right)= & N \iint \ldots \int\left[\Psi^{0}\left(\vec{x}, \vec{x}_{2}, \ldots, \vec{x}_{N}\right) \Psi^{I *}\left(\vec{x}^{\prime}, \vec{x}_{2}, \ldots, \vec{x}_{N}\right)\right]_{\sigma^{\prime} \rightarrow \sigma} \\
& d \vec{x}_{2} \ldots d \vec{x}_{N} d \sigma
\end{aligned}
$$

Here $\Psi^{0}$ stands for the $N$-electron ground-state wavefunction and $\Psi^{I}$ for the $N$-electron excited-state wavefunction of the $I$ th state. Both depend on $4 N$ variables -3 spatial $(\vec{r})$ and 1 spin variable $(\sigma)$ per electron collected in a vector $\vec{x}_{i}, i=1, \ldots, N$ (and parametrically 
on the nuclear geometry). The transition density is obtained from $\rho^{0 I}\left(\vec{r}, \vec{r}^{\prime}\right)$ by setting $\vec{r}^{\prime}=\vec{r}$.

In the framework of TDDFT (and TDDFTB as well) the groundstate wavefunction can be approximated by a single Slater determinant $\Phi$, which is composed of $N$ lowest energy Kohn-Sham molecular spin-orbitals $\chi_{i}(\vec{x})$ (this single Kohn-Sham Slater determinant is, in fact, an exact ground-state wavefunction of a system of non-interacting electrons with the same electron density as for the interacting system). The excited-state "wavefunction", in turn, can be thought of (in the spirit of configuration interaction singles) as a linear combination of singly-excited Stater determinants, $\Phi_{i}^{a}$, where a particular column corresponding to an occupied orbital $i$ is substituted with a column corresponding to a virtual (unoccupied in the ground state) orbital $a$ (having the same spin-function $\left[s_{i}(\sigma)=s_{a}(\sigma)\right]$, i.e., without spin flip):

$$
\Psi^{I}\left(\vec{x}_{1}, \vec{x}_{2}, \ldots, \vec{x}_{N}\right)=\sum_{i} \sum_{a} C_{i a}^{I} \Phi_{i}^{a}\left(\vec{x}_{1}, \vec{x}_{2}, \ldots, \vec{x}_{N}\right)
$$

The coefficients $C_{i a}^{I}$ are constructed from the solution of the linear response TDDFT(B) equations (see ref. 83 for details). We note that, strictly speaking, the concept of a wavefunction is alien to density functional theory. The "wavefunctions" should be understood merely as a convenient aid for computing transition densities and approximate non-adiabatic couplings.

Substituting expressions for the ground $\left(\Psi^{0}=\Phi\right)$ and excited-state (2) wavefunctions into (1) and using linear combinations of atomic orbitals $\eta(\vec{r})$ to represent molecular spin-orbitals $\chi(\vec{x})$ as $\chi_{i}(\vec{x})=$ $\sum_{\mu} c_{i \mu} \eta_{\mu}(\vec{r}) s_{i}(\sigma), \chi_{a}(\vec{x})=\sum_{\nu} c_{a \nu} \eta_{\nu}(\vec{r}) s_{a}(\sigma)$ we obtain

$$
\rho^{0 I}\left(\vec{r}, \vec{r}^{\prime}\right)=\sum_{\mu} \sum_{\nu} \sum_{i} \sum_{a} C_{i a}^{I *} c_{i \mu} c_{a \nu}^{*} \eta_{\mu}(\vec{r}) \eta_{\nu}^{*}\left(\vec{r}^{\prime}\right)
$$

Now we can define the matrix elements $P_{\mu \nu}$, which constitute the transition density matrix in the basis of atomic orbitals ${ }^{84}$

$$
P_{\mu \nu}=\sum_{i} \sum_{a} C_{i a}^{I *} c_{i \mu} c_{a \nu}^{*}
$$

The $P_{\mu \nu}$ matrix elements are central to the analysis of exciton dynamics studied in this work. This analysis is described in the next subsection. 


\subsection{Analysis of transition density localization}

Following the work of Tretiak and co-workers ${ }^{26,27}$ we, first, define two descriptors, which show the fraction of transition density (FTD) on a certain fragment $X(X=L$ (left), $M$ (middle), or $R$ (right) for the trimer under study, see Fig. 1a):

$$
\begin{gathered}
{ }^{1} \mathrm{FTD}_{X}=\frac{\sum_{\mu \in X} \sum_{\nu \in X} P_{\mu \nu}^{2}}{\sum_{\mu \in \text { molecule }} \sum_{\nu \in \text { molecule }} P_{\mu \nu}^{2}} \\
{ }^{2} \mathrm{FTD}_{X}=\frac{\sum_{\mu \in X} P_{\mu \mu}^{2}}{\sum_{\mu \in \text { molecule }} P_{\mu \mu}^{2}}
\end{gathered}
$$

The first descriptor ${ }^{1} \mathrm{FTD}_{X}$ accounts for all AOs belonging to the atoms of the fragment, whereas the second descriptor ${ }^{2} \mathrm{FTD}_{X}$ takes account of diagonal elements only. The fragments $L, M, R$ are defined as shown in Fig. 1a and b, each fragment comprises carbon atoms belonging to a single tetracene (hydrogens are not included). Since several excited states are involved in surface hopping calculations, we choose (in the spirit of the surface hopping approach) the transition density for a current electronic state at a given time $t$ as a quantity for analysis of exciton localization/delocalization at this time $t$.

Furthermore, we define highest $(\mathcal{H})$, intermediate $(\mathcal{I})$, and lowest $(\mathcal{L})$ monomers (fragments) at $0 \mathrm{fs}$ for each surface hopping trajectory, $\operatorname{FTD}_{\mathcal{H}}(0 \mathrm{fs})>\operatorname{FTD}_{\mathcal{I}}(0 \mathrm{fs})>\operatorname{FTD}_{\mathcal{L}}(0 \mathrm{fs})$, as well as highest, intermediate, and lowest monomers at current time $t, \operatorname{FTD}_{\mathcal{H}}(t)>\operatorname{FTD}_{\mathcal{I}}(t)>$ $\operatorname{FTD}_{\mathcal{L}}(t)$. We also define a participation number, $\mathrm{PN}=\left(\mathrm{FTD}_{L}^{2}+\right.$ $\left.\mathrm{FTD}_{M}^{2}+\mathrm{FTD}_{R}^{2}\right)^{-1}$. This quantity reflects the extent of exciton localization/delocalization over the fragments composing a molecule. In the case of complete delocalization between all 3 units (meaning that $\left.\mathrm{FTD}_{X} \approx 0.33 \forall X\right) \mathrm{PN}$ is close to 3 , while localization on a single unit $\left(\mathrm{FTD}_{X_{1}} \approx 1, \mathrm{FTD}_{X_{2,3}} \approx 0\right)$ corresponds to $\mathrm{PN} \approx 1$.

To extend the analysis of FTD we calculate the fraction of trajectories, having $\mathrm{FTD}_{X}$ in a certain interval $0.0+0.1 j<\mathrm{FTD}_{X} \leqslant$ $0.1+0.1 j(j=0,1,2, \ldots, 9)$ at a given time $t$. Constructing a relative frequency distribution from such fractions allows one to visualize the extent of spatial localization/delocalization of the exciton at a fixed time. Specifically, large peaks at small and big values of $\mathrm{FTD}_{X}$ correspond to localization of the exciton. Another characteristic, which 
we introduce, is the fraction of trajectories, possessing an FTD gap, $\max _{t}\left(\mathrm{FTD}_{X}\right)-\min _{t}\left(\mathrm{FTD}_{X}\right)$ (defined for each trajectory separately), in a certain interval $0.0+0.1 j<\max _{t}\left(\mathrm{FTD}_{X}\right)-\min _{t}\left(\mathrm{FTD}_{X}\right) \leqslant$ $0.1+0.1 j(j=0,1,2, \ldots, 9)$. Large values of this gap indicate that during a nonadiabatic molecular dynamics run, $\mathrm{FTD}_{X}$, localized on a given monomer $X$, experiences substantial changes, e.g., the excitation can "travel away" or, oppositely, "visit" the fragment of interest. In addition, we define large sudden changes of $\mathrm{FTD}_{X}, \Delta \mathrm{FTD}_{X}=\mathrm{FTD}_{X}(t+$ $(1 \mathrm{fs}))-\mathrm{FTD}_{X}(t)>0.5$. These changes represent abrupt jumps occurring at the ultra-short time of $1 \mathrm{fs}$. We note that the number 0.5 on the right-hand side is somewhat arbitrary. The ultrafast abrupt FTD changes may happen when a certain change of nuclear configuration occurs. The fraction of large sudden changes $\Delta \mathrm{FTD}_{X}>0.5$ in a certain time interval $(0+50 j) \mathrm{fs} \leqslant t<(50+50 j)$ fs $(j=0,1,2, \ldots, 9)$ as well as the fraction of trajectories having $n$ large sudden changes during the course of excited-state dynamics, are also computed in this work.

\section{Results and discussion}

The most intense electronic transition (among transitions to low-lying states) is the $S_{0} \rightarrow S_{1}$ transition, as determined with long-range corrected TDDFT and TDDFTB calculations, as well as with the other tested approaches. The excitation energies and oscillator strengths calculated for this transition with different methods are compared in Tab. 1. 
Table 1: Excitation energies, wavelengths, and oscillator strengths for the $S_{0} \rightarrow S_{1}$ transition of the tetracene trimer computed with various methods at the $\omega$ B97X-D/def2-TZVP optimized geometry. For the long-range corrected TDDFTB method (LC-TDDFTB) the size of active space (number of highest occupied $\times$ number of lowest virtual orbitals) is shown in parentheses

\begin{tabular}{lccc}
\hline Method & $\Delta E(\mathrm{eV})$ & $\lambda(\mathrm{nm})$ & $f$ \\
\hline ADC $(2) / \mathrm{cc}-p V D Z$ & 2.90 & 428 & 0.67 \\
AM1/CISDT $(12 \times 12)$ & 2.92 & 425 & 0.88 \\
HF/def2-TZVP & 2.90 & 427 & 0.93 \\
B3LYP/6-31G** & 2.46 & 505 & 0.24 \\
CAM-B3LYP/6-31G** & 2.82 & 440 & 0.67 \\
CAM-B3LYP/def2-TZVP & 2.71 & 458 & 0.59 \\
$\omega$ B97/def2-TZVP & 2.93 & 423 & 0.77 \\
$\omega$ B97X/def2-TZVP & 2.87 & 432 & 0.72 \\
$\omega$ B97X-D /def2-TZVP & 2.75 & 450 & 0.63 \\
LC-TDDFTB $($ full, $180 \times 180)$ & 2.50 & 496 & 0.58 \\
LC-TDDFTB $(100 \times 100)$ & 2.64 & 470 & 0.67 \\
LC-TDDFTB $(50 \times 50)$ & 2.72 & 455 & 0.73 \\
LC-TDDFTB $(30 \times 30)$ & 2.76 & 450 & 0.74 \\
LC-TDDFTB $(20 \times 20)$ & 2.83 & 438 & 0.78 \\
LC-TDDFTB $(10 \times 10)$ & 2.94 & 422 & 0.56 \\
\hline
\end{tabular}

From Tab. 1 and from Fig. 1c we see that the excitation energies for the $S_{0} \rightarrow S_{1}$ transition agree remarkably well between TD- $\omega \mathrm{B} 97 \mathrm{X}$ $\mathrm{D} /$ def2-TZVP and LC-TDDFTB $(30 \times 30)$. The latter is used for the dynamics in what follows. Interestingly, the excitation energy of the $S_{0} \rightarrow S_{1}$ transition computed with LC-TDDFTB using the full actve space is closer to that obtained with B3LYP rather than those calculated using the range-separated functionals. The contraction of the active orbital space leads to a blue shift of the absorption spectrum. The oscillator strengths are also similar between TD- $\omega$ B97X-D/def2TZVP and LC-TDDFTB $(30 \times 30)$. The excitation corresponding to the $S_{0} \rightarrow S_{1}$ transition (considering the optimized $\omega \mathrm{B} 97 \mathrm{X}-\mathrm{D} /$ def2TZVP geometry) is somewhat delocalized over 3 fragments of the trimer, as can be seen from natural transition orbital (NTO) analysis ${ }^{85}$ of the TD- $\omega$ B97X-D/def2-TZVP and LC-TDDFTB results (see Fig. 2a and b). 

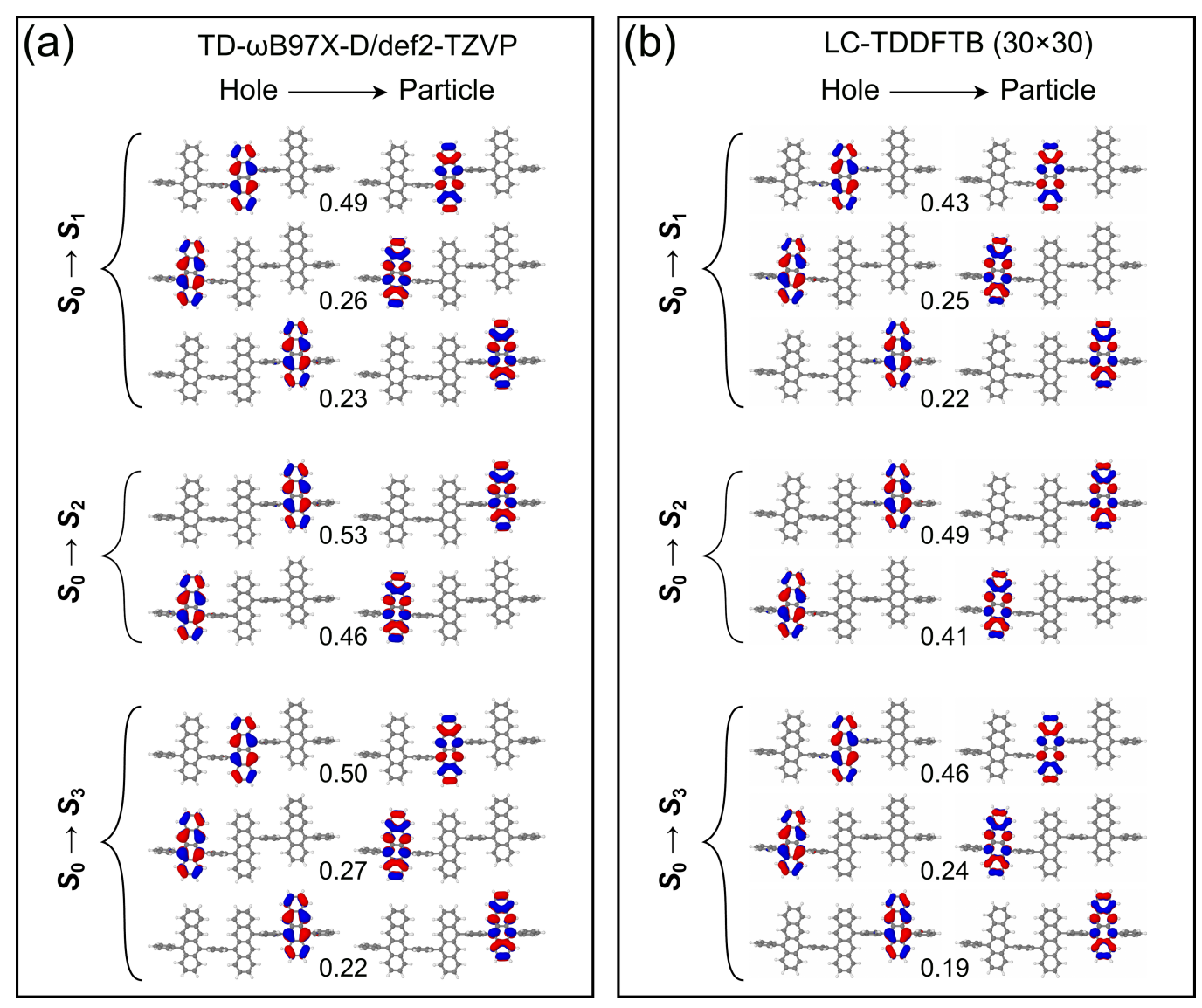

Figure 2: Dominant natural transition orbital (NTO) pairs for the $S_{0} \rightarrow$ $S_{1}, S_{0} \rightarrow S_{2}$ and $S_{0} \rightarrow S_{3}$ transitions calculated at the (a) $\omega$ B97X-D/def2TZVP and (b) LC-TDDFTB $(30 \times 30)$ levels of theory. The number below each pair is the corresponding NTO contribution to the transition. The geometry optimized at the $\omega$ B97X-D/def2-TZVP level is used (see Fig. 1a).

The middle fragment accumulates $\sim 50 \%$ of the excitation, whereas the outer fragments share almost equally the other $\sim 50 \%$. We note that the NTO analysis allows one to simplify the characterization of excited states, compared to the inspection of transitions between conventional molecular orbitals. The latter, however, may also provide a valuable insight into, e.g., the polaron delocalization. ${ }^{86}$ The NTOs for the second and the third transitions are also presented in Fig. 2a and b. These transitions, with oscillator strength of $\sim 0$ (see Fig. 1c), correspond to higher lying exciton states associated with the bright 
$\pi \rightarrow \pi^{*}$ transition in tetracene. The difference between TD- $\omega$ B97X$\mathrm{D} /$ def2-TZVP and LC-TDDFTB $(30 \times 30)$ excitation energies for the $S_{0} \rightarrow S_{2}$ and $S_{0} \rightarrow S_{3}$ transitions is more pronounced than for the $S_{0} \rightarrow S_{1}$ transition (Fig. 1c). In particular, the energy separation of $S_{3}$ from $S_{2}$ is smaller on the TD- $\omega$ B97X-D/def2-TZVP level than on the LC-TDDFTB $(30 \times 30)$ level, $\sim 0.03$ vs. $\sim 0.08 \mathrm{eV}$, respectively. When using the LC-TDDFTB with the full active space the $S_{2}-S_{3}$ energy gap becomes $\sim 0.04 \mathrm{eV}$. Thus, the contraction of the active space affects the energy separation of electronic states. Nevertheless, the differences are on the order of $0.1 \mathrm{eV}$, which, we believe, is acceptable for the semiempirical treatment. Notably, the LC-TDDFTB NTO contributions are highly correlated with the respective LC-TDDFT NTO contributions (compare numbers in Fig. 2a to those in Fig. 2b).

Table 2: Energy gap between the $S_{3}$ and $S_{4}$ states calculated at different levels of theory for the $\omega \mathrm{B} 97 \mathrm{X}-\mathrm{D} /$ def2-TZVP optimized geometry

\begin{tabular}{lc}
\hline Method & $S_{3}-S_{4}$ gap $(\mathrm{eV})$ \\
\hline LC-TDDFTB $(30 \times 30)$ & 0.39 \\
LC-TDDFTB (full, $180 \times 180)$ & 0.59 \\
TD- $\omega$ B97X-D /def2-TZVP & 0.87 \\
ADC $(2) /$ cc-pVDZ & 0.64 \\
\hline
\end{tabular}

We also note that the B3LYP calculation demonstrates the admixture of spurious CT excitations into the local ones, what is reflected in much smaller oscillator strength of the $S_{0} \rightarrow S_{1}$ transition (Tab. 1). In TDDFTB without LC the lowest transitions are also of the CT character. Thus the use of LC (or large fraction of exact exchange) is necessary for the correct description of the nature of the low-lying excited states of the tetracene trimer under study.

In order to estimate the energies of the doubly-excited states which presumably play a role in the intramolecular singlet fission, ${ }^{56}$ we have calculated the lowest excited states of the trimer using quasidegenerate perturbation theory with general multiconfigurational reference functions. The three lowest excited states $\left(S_{1}-S_{3}\right.$, with excitation energies $2.30,2.35$, and $2.42 \mathrm{eV}$, respectively) can be classified as the singlyexcited states whereas the next three states $\left(S_{4}-S_{6}\right.$, with excitation energies $2.72,2.72$, and $2.74 \mathrm{eV}$, respectively), which are $\sim 0.3 \mathrm{eV}$ higher than the $S_{3}$ state, have dominantly doubly-excited character. Thus, we assume that these doubly-excited states will not substantially 
affect the short-time dynamics of the trimer.

The broadened absorption spectrum, obtained from vertical absorption spectra calculated with the LC-TDDFTB $(30 \times 30)$ method at the geometries sampled from the LC-DFTB ground-state molecular dynamics trajectory (Fig. 1b) is overall close (in excitation energies) to the experimental one (see Fig. 1c). The calculated spectrum spans the range $\sim 370-480 \mathrm{~nm}$, the experimental one $\sim 400-510 \mathrm{~nm}$. Three remarks should be made here: (i) The experimental spectrum was recorded in toluene, whereas calculations are performed for the gas phase. The environment can in general cause spectral shifts. (ii) The experimental spectrum includes vibrational progression, whereas the different bands of the calculated spectrum reflect difference in vertical electronic spectra, which in turn differ due to classical description of nuclear motion. (iii) The LC correction may also lead to the blue shift, as well as the reduced orbital active space (see Tab. 1). Although all these observations make exact comparison not possible, the difference in absorption maxima between theory and experiment of $\sim 0.3 \mathrm{eV}$ indicates that the semiempirical LC-TDDFTB $(30 \times 30)$ method describes absorption rather well.

To unveil the effect of coupling between electron and nuclear dynamics on the molecular exciton we perform nonadiabatic simulations. As mentioned in section 2, the 3 lowest excited states and the ground state are included in the surface hopping simulations. We choose 3 excited states based on rather large $S_{3}-S_{4}$ energy gap. This gap calculated using LC-TDDFTB, LC-TDDFT and ADC(2) methods is shown in Tab. 2. It is seen that this gap is $\sim 0.4 \mathrm{eV}$ when using LC-TDDFTB $(30 \times 30)$ and more than $0.5 \mathrm{eV}$ with all other methods. Moreover, the first three excited states of the trimer arise naturally in the framework of molecular exciton theory. ${ }^{5,6}$

Fig. 3 shows the electronic state populations, i.e., fractions of trajectories, running in a particular state $S_{I}$ at a time $t$ obtained from surface hopping simulations. 


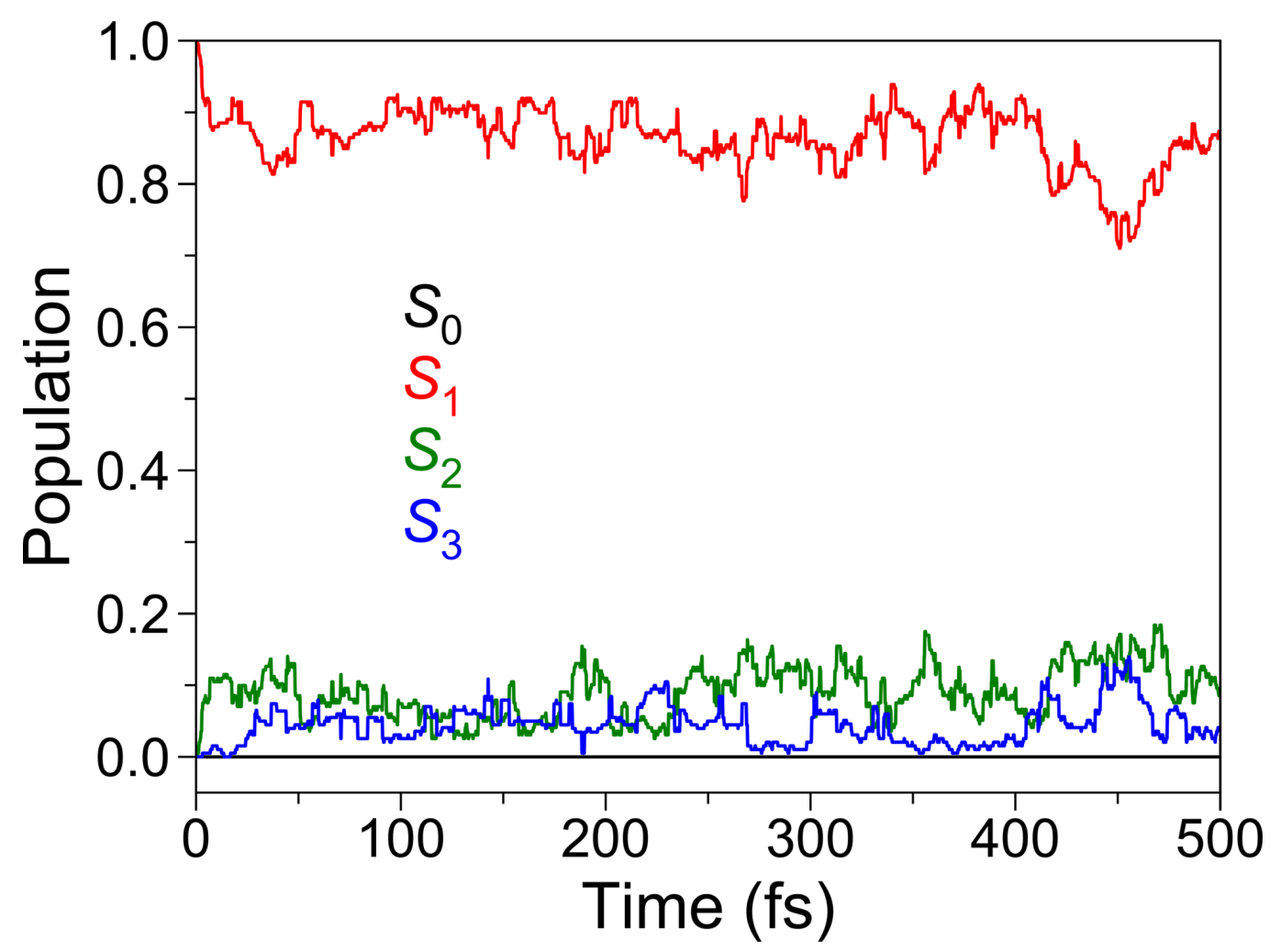

Figure 3: Evolution of populations (fractions of trajectories) for the ground $\left(S_{0}\right)$ and excited $\left(S_{1}, S_{2}\right.$, and $\left.S_{3}\right)$ states obtained from LC-TDDFTB nonadiabatic dynamics simulations.

After photoexcitation to $S_{1}$, the trimer remains mostly in this electronic state throughout all 500 fs of the simulation. The $S_{2}$ and $S_{3}$ states are weakly populated. Notably, the population of $S_{0}$ is exactly zero, i.e., there is no non-radiative decay from $S_{1}$ to $S_{0}$, at least during the first $500 \mathrm{fs}$.

Although the molecule resides in the $S_{1}$ state for the whole simulation period (on average), the exciton localization/delocalization picture can vary with time, owing to an effect of nuclear motion on the diabatic character of the electronic states. To address this question we analyze transition density localization/delocalization over molecular fragments (defined in Fig. 1a). The time evolutions of the averaged FTDs over all 115 trajectories for all 3 fragments are shown in Fig. 4a. 

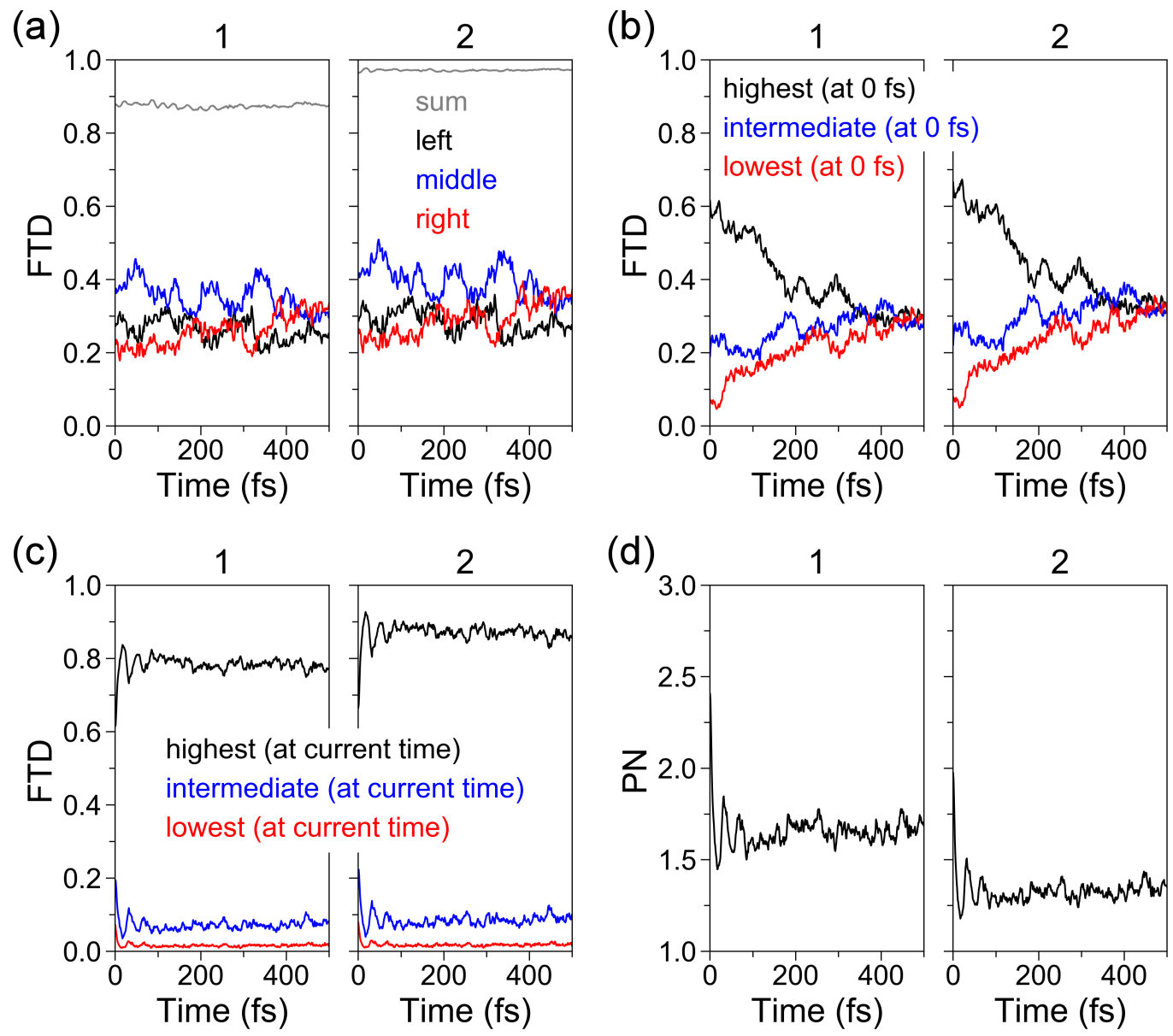

Figure 4: (a) Evolution of the fraction of transition density (FTD) belonging to different fragments (as well as the sum of fractions). (b) Evolution of FTD belonging to the highest, intermediate, and lowest monomers, defined at 0 fs. (c) Evolution of FTD belonging to the highest, intermediate, and lowest monomers, defined at current time $t$. (d) Participation number (PN) as a function of time. The plots with the title "1" correspond to the ${ }^{1} \mathrm{FTD}_{X}$ descriptor whereas the plots with the title "2" correspond to ${ }^{2} \mathrm{FTD}_{X}$.

First of all, we note that the averaged FTDs for different fragments are comparable to each other, and amount to about 0.3. At the beginning, the middle fragment dominates over the right and the left ones (bearing resemblance to the results obtained for the $\omega \mathrm{B} 97 \mathrm{X}$ $\mathrm{D} /$ def2-TZVP geometry optimized in the ground state, see Fig. 2a 
and b), but this picture changes to some extent towards the end of the simulation (500 fs), where all three FTDs merge and correspond to the value $\sim 0.3$. In Fig. 4 a we present both types of FTDs, ${ }^{1} \mathrm{FTD}_{X}$ and ${ }^{2} \mathrm{FTD}_{X}$. It is seen that both descriptors provide similar trends of FTD variation with time. We note that ${ }^{2} \mathrm{FTD}_{X}$ is larger than ${ }^{1} \mathrm{FTD}_{X}$, what is also reflected by the sum over fragments: The sum ${ }^{1} \mathrm{FTD}_{L}+{ }^{1} \mathrm{FTD}_{M}+{ }^{1} \mathrm{FTD}_{R} \approx 0.9$, whereas ${ }^{2} \mathrm{FTD}_{L}+{ }^{2} \mathrm{FTD}_{M}+{ }^{2} \mathrm{FTD}_{R}$ is closer to 1 . The rest of FTD resides at the atoms that do not belong to fragments $L, M, R$ (and also includes weak charge-transfer processes between fragments, in case of the ${ }^{1} \mathrm{FTD}_{X}$ descriptor).

Thus, Fig. 4a demonstrates that on average all three fragments bear $\sim 0.3$ of FTD, not much dependent on time. However, Fig. 4a is not able to answer the question of how this mean values are brought about, whether by delocalization or localization of the molecular exciton. Indeed, the mean value of $\sim 0.3$ can be obtained, e.g., if all trajectories exhibit the full delocalization of the exciton over all 3 fragments. Oppositely to this, the same mean value of $\sim 0.3$ will be obtained if a third of all trajectories have the exciton localized only on the left fragment, the next third on the middle fragment, and the last third on the right fragment.

Therefore we perform further analysis of the FTDs, in terms of highest, intermediate, and lowest fragments, as defined above. Fig. 4b depicts the FTD time evolution for $\mathcal{H}, \mathcal{I}$, and $\mathcal{L}$ fragments, defined at time $t=0 \mathrm{fs}$. Here we immediately notice that in the beginning of the simulation the values of FTD for these 3 types of fragments are quite different from each other. Namely, the highest fragment has an average value of about 0.6 , while the lowest one acquires $\sim 0.1$; the intermediate monomer has about 0.2 . In other words, ground-state molecular dynamics leads to certain exciton localization in the trimer, in comparison to the results for the single optimized geometry presented in Fig. $2 \mathrm{a}$ and $\mathrm{b}$. The conformational disorder induced by molecular motion at $300 \mathrm{~K}$ (in the electronic ground state) leads to redistribution of electronic transition density in comparison to the minimum of the ground-state potential energy surface.

Notably, towards the end of the simulation period (500 fs) all 3 FTDs in Fig. 4b merge with each other. This clearly demonstrates that the fragment having the highest FTD at the beginning will not always serve as the $\mathcal{H}$ fragment throughout the simulation, but rather the other fragments can take this role or complete delocalization can take place, meaning that all 3 fragments have equal FTDs of about 


\section{3 .}

To elaborate on the problem of localization/delocalization further we analyze the FTD evolution for $\mathcal{H}, \mathcal{I}$, and $\mathcal{L}$ fragments, defined at the current time $t$ (individually for each trajectory) (Fig 4c). The starting values (at $t=0 \mathrm{fs}$ ) of FTD are the same as in Fig. 4b. However, the subsequent evolution differs drastically from that of Fig. 4b: The highest monomer gains more TD in ultra-short time, while the lowest and intermediate monomers lose the TD. Specifically, the maximum in $\mathrm{FTD}_{\mathcal{H}}$ is observed at $17 \mathrm{fs}$. This result provides the evidence for ultrafast exciton localization during excited-state dynamics of the tetracene trimer. This finding is further corroborated by the time evolution of the participation number (Fig. 4d). PN falls rapidly and reaches its minimum at $17 \mathrm{fs}$. As already mentioned above, the decrease of participation number indicates localization of a molecular exciton. Indeed, the fraction of trajectories, having small and large values of $\mathrm{FTD}_{X}$ increases in comparison to the beginning (see Fig. 5). 

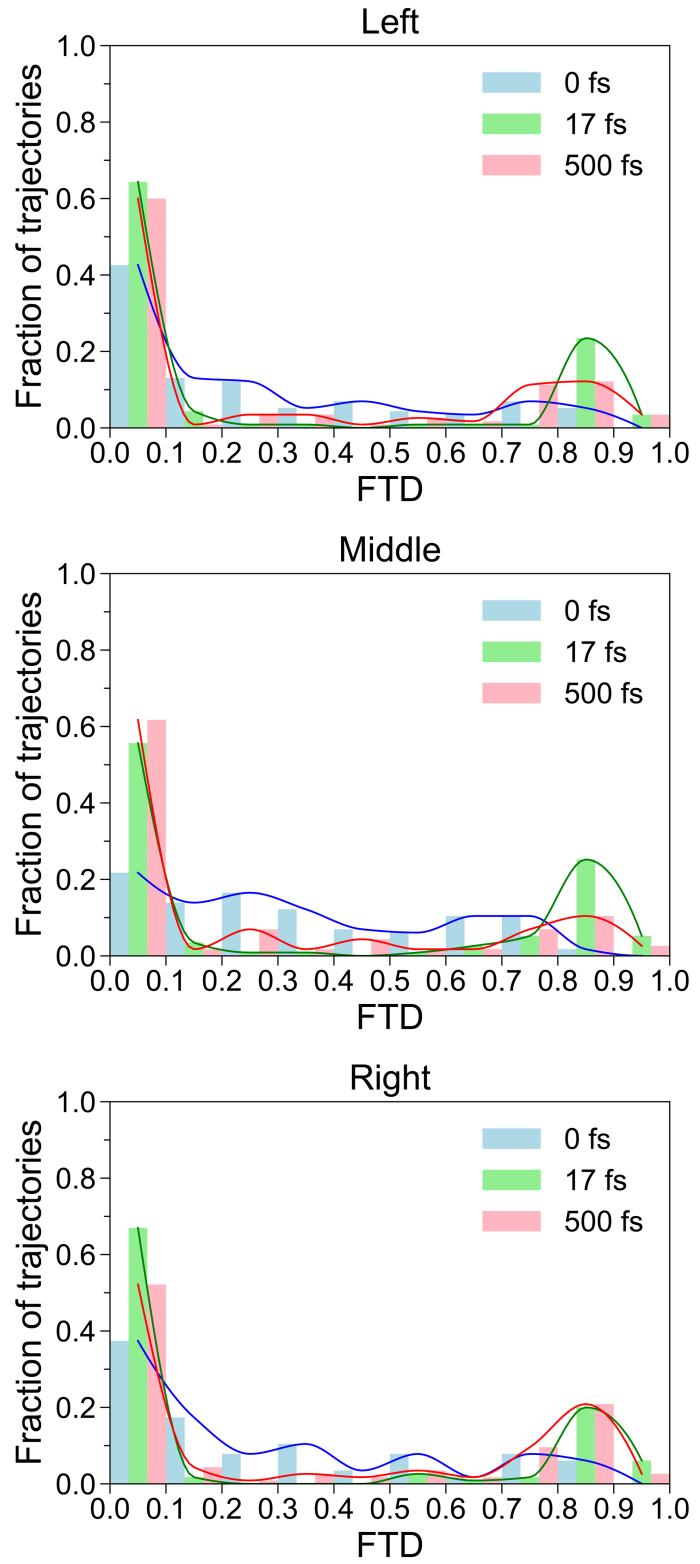

Figure 5: Fraction of trajectories, having a certain fraction of transition density $0.0+0.1 j<{ }^{1} \operatorname{FTD}_{X} \leqslant 0.1+0.1 j(j=0,1,2, \ldots, 9)$, for various times. Curves are interpolated results, presented to clarify the figure.

Remarkably, at time 17 fs there are almost no trajectories with $0.2<{ }^{1} \mathrm{FTD}_{X} \leqslant 0.4$, i.e., corresponding to the range of FTD associated 
with large delocalization. In the end of the simulation the exciton is somewhat more delocalized than at 17 fs.

One comment is in place here. Taking a look at the distributions of Fig. 5 for a time of $17 \mathrm{fs}$, we realize that roughly $60-70 \%$ of the trajectories show very small FTD for a given, geometrically-defined fragment (either $L$, or $M$, or $R$ ), whereas $20-30 \%$ of trajectories correspond to strong localization of TD on the fragment (either $L$, or $M$, or $R$ ). Thus, when looking at the swarm of trajectories at a given time (17 fs) the exciton is located on all 3 fragments. However, there is no "true" delocalization of excitation, i.e., for each single geometry from the swarm, over all 3 fragments.

The initial oscillations of FTD for $\mathcal{H}, \mathcal{I}, \mathcal{L}$ monomers defined at the current time as well as PN are correlated with changes in $\mathrm{CC}$ bond lengths of the molecule during excited-state dynamics (Fig. 6).

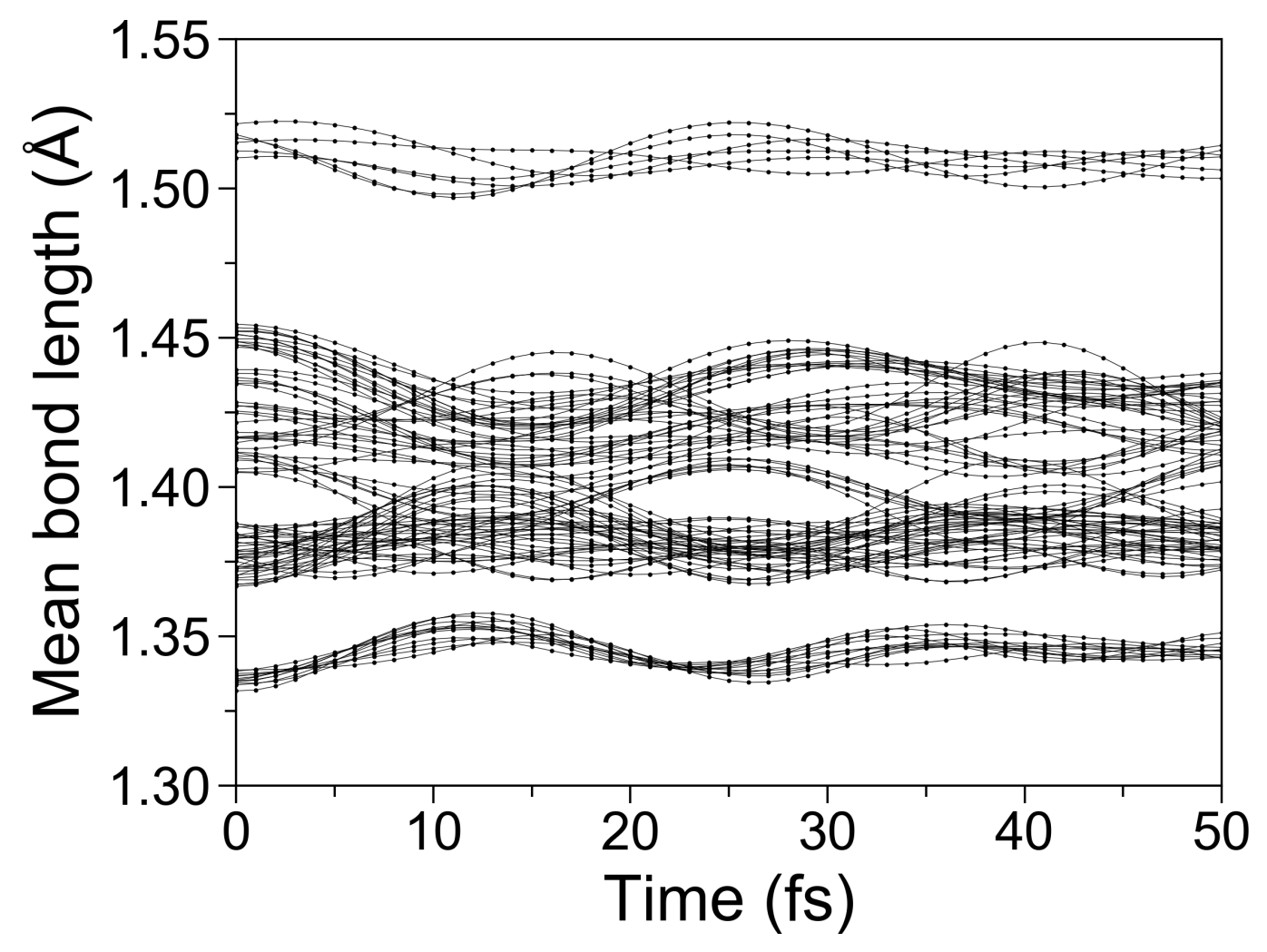

Figure 6: Time evolution of the mean (over ensemble of trajectories) bond lengths for all $93 \mathrm{CC}$ bonds of the trimer. 
Many bond length evolutions exhibit a first minimum or maximum between 10 and $20 \mathrm{fs}$, while the maximum in localization is reached at 17 fs (see Figs. 4c and d).

Another intriguing question refers to the dynamics of exciton localization. More precisely, how this localization is maintained, either through sudden jumps of the exciton from one fragment to another, or gradual changes between localized states, meaning transient delocalization over fragments. To elucidate this problem we plot the time evolutions of ${ }^{1} \mathrm{FTD}_{X}$ for all 115 trajectories at once (Fig. 7), using scatter plots. 

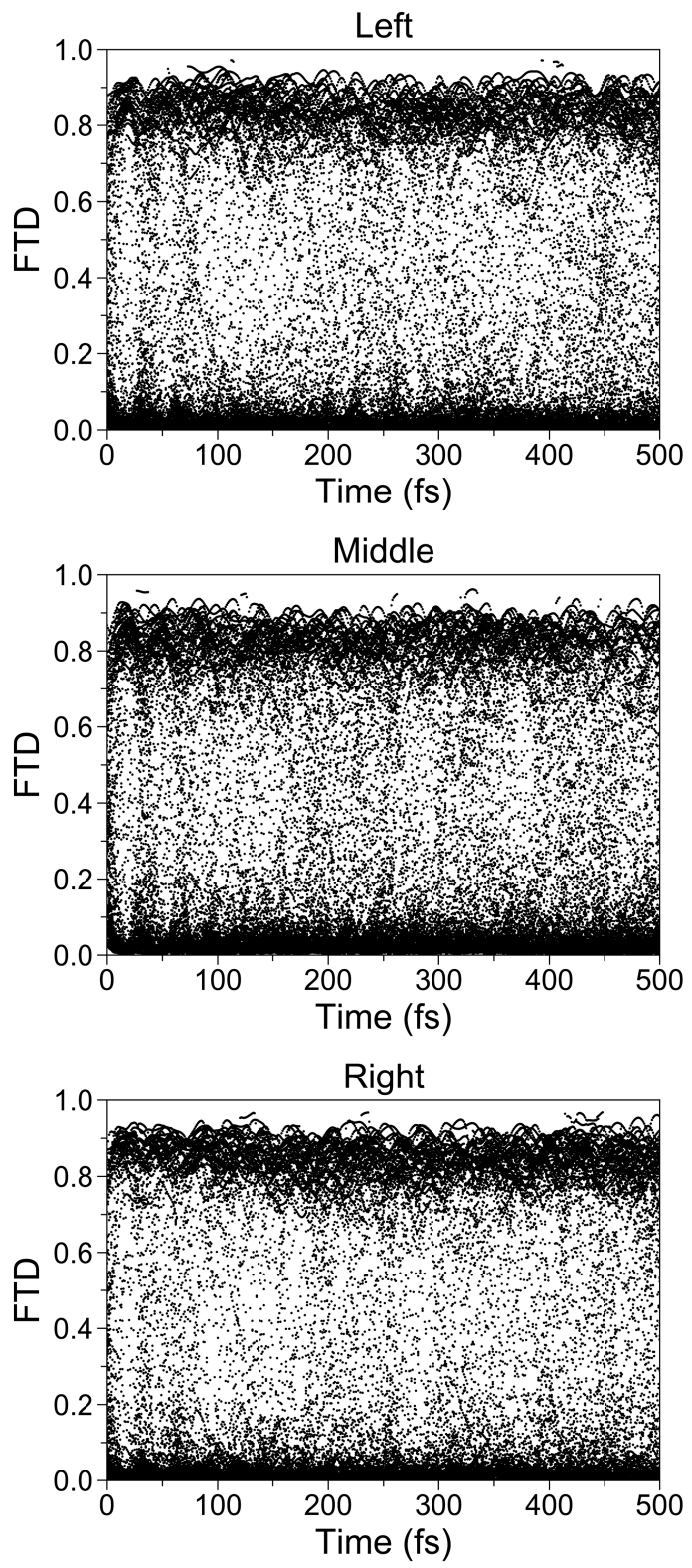

Figure 7: Evolution of ${ }^{1} \mathrm{FTD}_{X}$ for all fragments and all trajectories.

Here two observations can be made. First, the density of points (along the vertical axis) is large at low as well as high values of FTD. Second, there are points in the range between areas of high density (although the density of these points is much smaller). The first obser- 
vation indicates the localization of the exciton on a specific fragment, while the second one provides evidence for transient exciton delocalization, at a single-trajectory level. To facilitate presentation we provide a video (see SI) for a single trajectory, demonstrating the time evolution of the spatial representation of the transition density in parallel with the time evolution of the ${ }^{1} \mathrm{FTD}_{X}$ descriptor. We note that for this particular trajectory no surface hops were detected, the current electronic state is $S_{1}$. Thus, the dynamics is adiabatic for this trajectory. Despite of this, the exciton localization and the exciton transfer are observed in this case as can be seen from the video. The change in ${ }^{1} \mathrm{FTD}_{X}$ for this single particular trajectory along with the snapshots featuring ultrafast exciton localization to a single unit with subsequent transient delocalization and exciton transfer between units are also presented in Fig. 8. 




Figure 8: Time evolution of ${ }^{1} \mathrm{FTD}_{X}$ for the single trajectory along with the snapshots of transition density demonstrating ultrafast exciton localization to a single unit, transient exciton delocalization over fragments, and exciton transfer between units. Current state is $S_{1}$ throughout dynamics.

The large density of points at low and high FTD values for a given 
fragment $X$ (Fig. 7) can, in principle, mean that some trajectories are confined either to high or low FTD values throughout the simulation. The opposite possibility corresponds to a large FTD change between low and high values during dynamics, at a single-trajectory level. To distinguish between these two scenarios we count the trajectories, possessing a certain FTD gap, defined as $\max _{t}\left(\mathrm{FTD}_{X}\right)-\min _{t}\left(\mathrm{FTD}_{X}\right)$. Here the maximum and the minimum (both over time) are searched for each trajectory individually. The obtained histograms are shown in Fig. 9. 

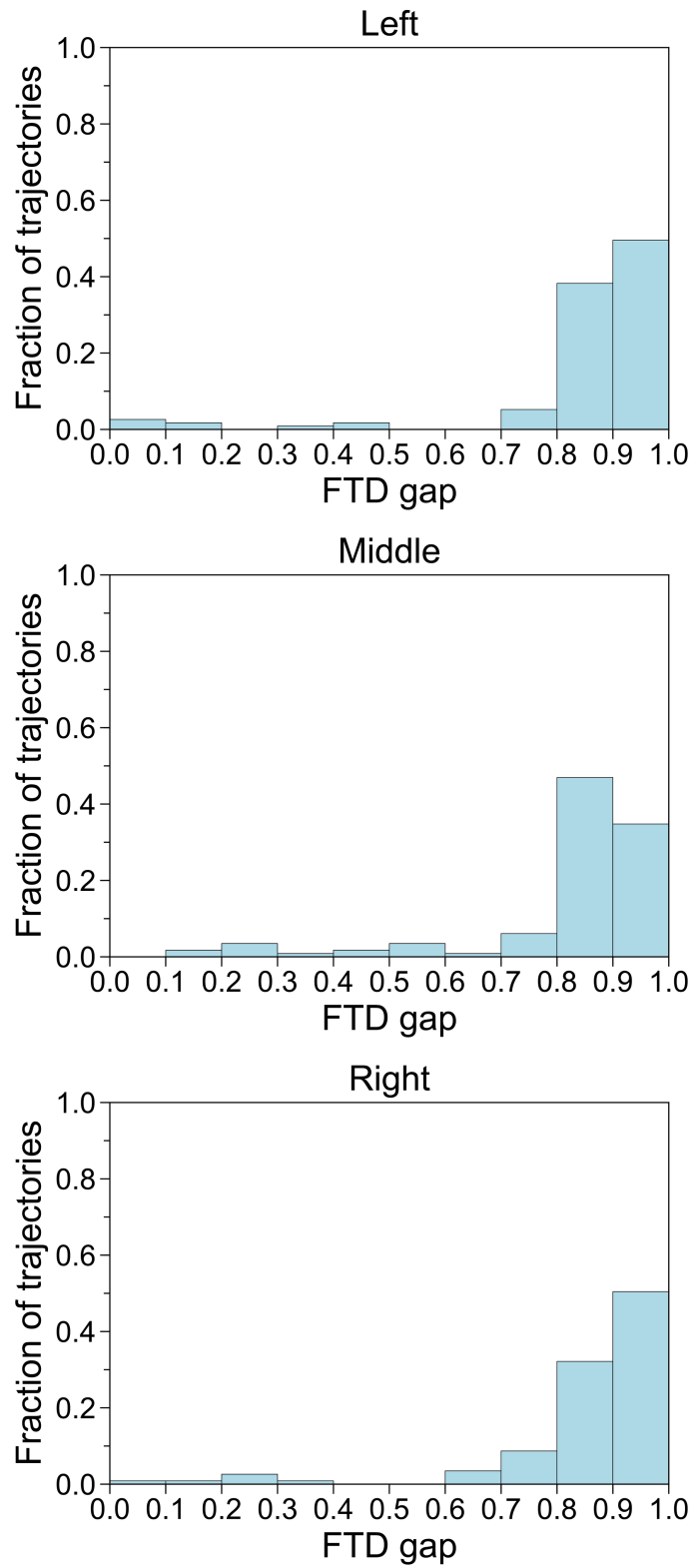

Figure 9: Fraction of trajectories, having a certain FTD gap $0.0+0.1 j<$ $\max _{t}\left({ }^{1} \mathrm{FTD}_{X}\right)-\min _{t}\left({ }^{1} \mathrm{FTD}_{X}\right) \leqslant 0.1+0.1 j(j=0,1,2, \ldots, 9, X=L, M, R)$.

As can be seen, the vast majority of trajectories demonstrate the large change in $\mathrm{FTD}_{X}$ during 500 fs of excited-state dynamics. Thus, during excited-state dynamics molecular fragments exchange the exci- 
ton between each other.

We note that for an idealized case of localization, corresponding to sudden jumps of the exciton, there would be no points at intermediate values of FTD (cf. Fig. 7). "Sudden" here refers to the time scale of 1 fs. To clarify the emerged picture of exciton dynamics further, we analyze the number of large sudden changes of FTD. These large sudden changes are somewhat arbitrarily defined to be greater than 0.5 on a time step of $1 \mathrm{fs}$. Fig. 10a demonstrates that none or only a few large sudden FTD changes are usually observed for a single surface hopping trajectory, while large numbers of large sudden changes are disfavoured. 
(a)
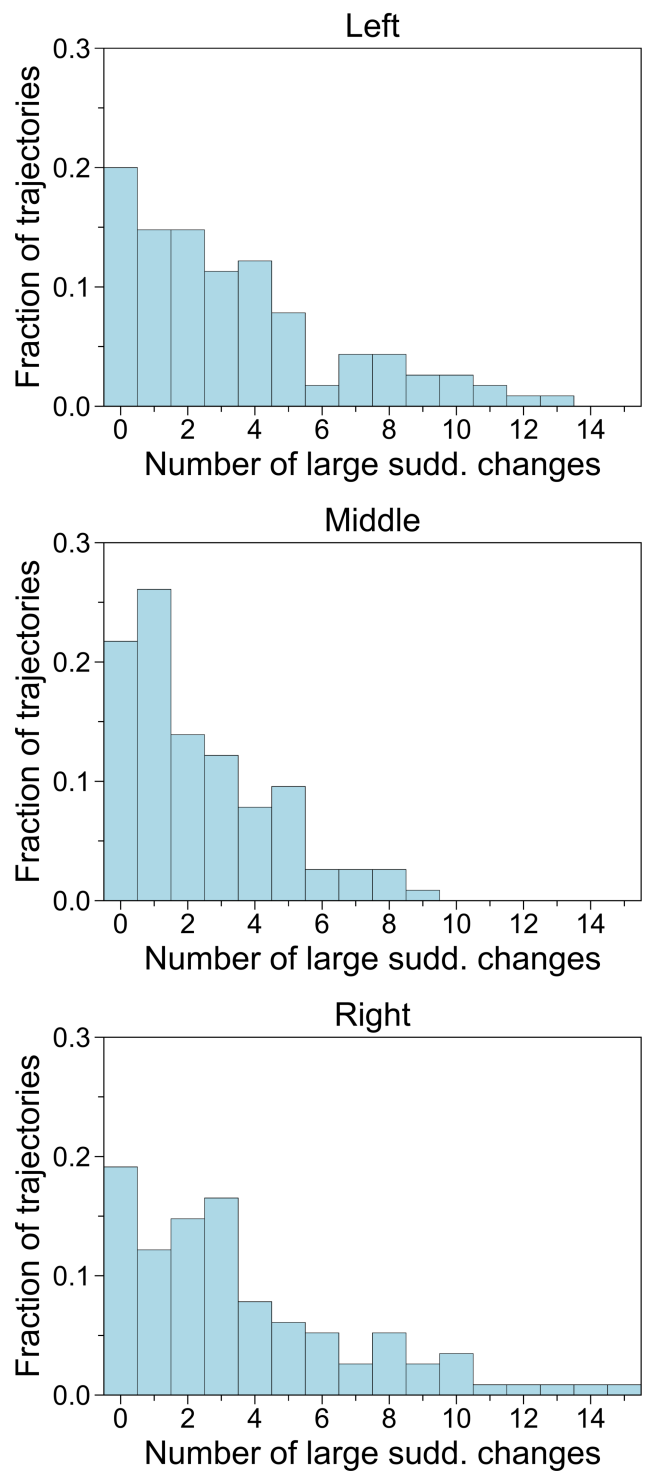

(b)
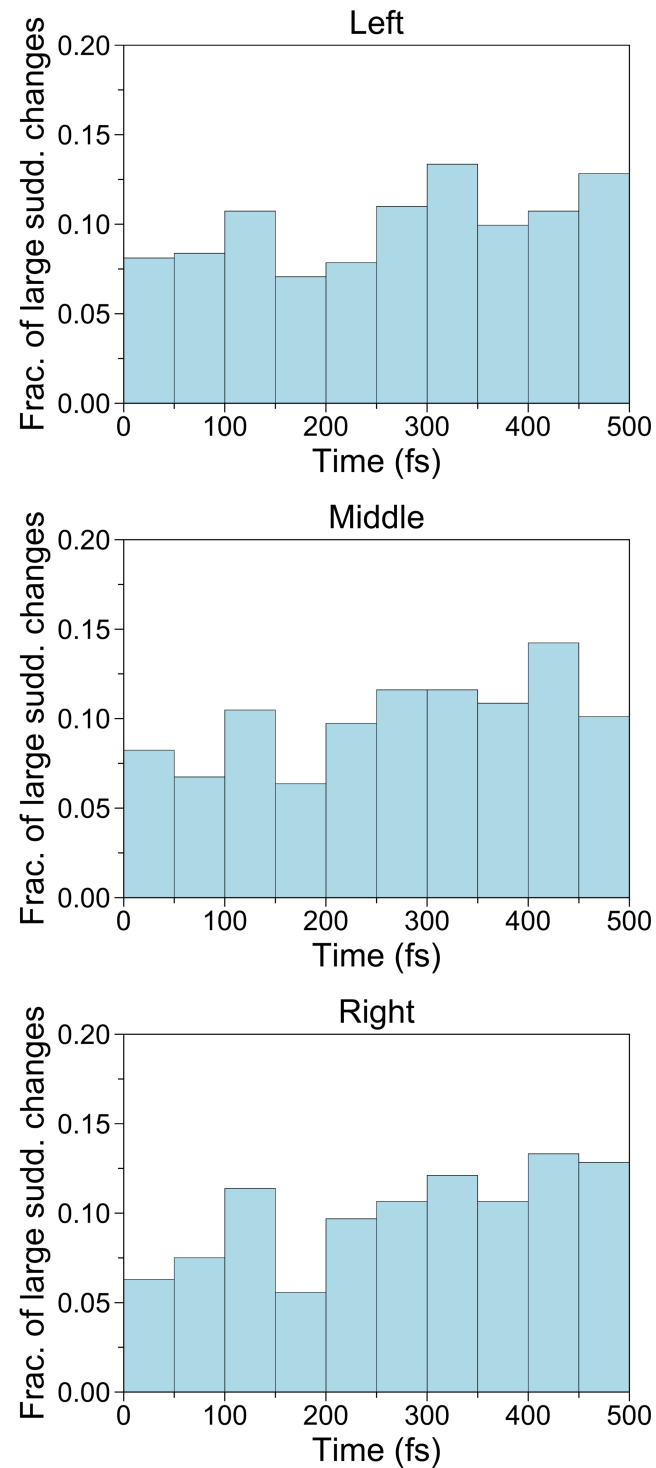

Figure 10: Fraction of trajectories, having certain number of large sudden ${ }^{1} \mathrm{FTD}_{X}$ changes. (b) Fraction of large sudden ${ }^{1} \mathrm{FTD}_{X}$ changes occurring within certain time interval, $(0+50 j)$ fs $\leqslant t<(50+50 j)$ fs $(j=0,1,2, \ldots, 9)$.

When occurring, these abrupt jumps of the exciton can happen at any time of simulation and they are quasi uniformly distributed in 
time (Fig. 10b).

The fact that almost all trajectories possess the large FTD gap (Fig. 9) along with the observation that large sudden changes are not favoured (Fig. 10a) verify the transient exciton delocalization in the course of excited-state dynamics, as a dominating mechanism for the exciton transfer.

\section{Conclusions}

In this work we addressed the singlet exciton dynamics of the recently synthesized covalently-linked tetracene trimer. Namely, we performed long-range corrected linear response TDDFTB surface hopping dynamics simulations of excited-state molecular dynamics of this trimer with subsequent comprehensive analysis of exciton localization by means of transition density matrix.

After excitation of the $S_{1}$ electronic state the molecule mostly remains in this state throughout 500 fs of simulation, with slight population of upper excited states and no return to the ground state.

Remarkably, excited-state dynamics leads to ultrafast exciton localization to a single tetracene unit of the trimer, happening during the first $17 \mathrm{fs}$. This localized picture is then preserved to high extent for the whole period of the simulation. Either of the fragments (left, middle, or right) can serve as a host for the exciton. These fragments exchange the exciton between each other during the course of the excited-state dynamics. This exchange, in turn, occurs mainly through transient exciton delocalization over the fragments. The large sudden (on a time scale of $1 \mathrm{fs}$ ) exciton jumps also happen, but are minor, as shown by statistical analysis including all the surface hopping trajectories.

\section{Acknowledgment}

E.T and R.M. are grateful to the Deutsche Forschungsgemeinschaft (DFG) for financial support through "FOR 1809: Light-induced Dynamics in Molecular Aggregates". A.H. and R.M. acknowledge funding by the European Research Council (ERC) Consolidator Grant DYNAMO (Grant No. 646737). E.T. thanks Joscha Hoche and Prof. Thibaud Etienne for valuable discussions. 


\section{Conflict of interest}

There are no conflicts to declare.

\section{References}

1. G. Scholes and G. Rumbles, Nat. Mater., 2006, 5, 683-696.

2. T. Brixner, R. Hildner, J. Köhler, C. Lambert and F. Würthner, Adv. Energy Mater., 2017, 7, 1700236.

3. T. Mirkovic, E. E. Ostroumov, J. M. Anna, R. van Grondelle, Govindjee and G. D. Scholes, Chem. Rev., 2017, 117, 249-293.

4. G. J. Hedley, A. Ruseckas and I. D. W. Samuel, Chem. Rev., 2017, 117, 796-837.

5. A. S. Davydov, Soviet Physics Uspekhi, 1964, 7, 145.

6. M. Kasha, H. Rawls and M. El-Bayoumi, Pure Appl. Chem., 1965, 11, 371-392.

7. N. J. Hestand and F. C. Spano, Acc. Chem. Res., 2017, 50, 341350 .

8. E. Titov and P. Saalfrank, J. Phys. Chem. A, 2016, 120, 30553070 .

9. E. Titov, G. Granucci, J. P. Götze, M. Persico and P. Saalfrank, J. Phys. Chem. Lett., 2016, 7, 3591-3596.

10. T. Nelson, S. Fernandez-Alberti, A. E. Roitberg and S. Tretiak, J. Phys. Chem. Lett., 2017, 8, 3020-3031.

11. T. Nelson, S. Fernandez-Alberti, A. E. Roitberg and S. Tretiak, Acc. Chem. Res., 2014, 47, 1155-1164.

12. A. Schubert, V. Settels, W. Liu, F. Würthner, C. Meier, R. F. Fink, S. Schindlbeck, S. Lochbrunner, B. Engels and V. Engel, J. Phys. Chem. Lett., 2013, 4, 792-796.

13. R. Binder, D. Lauvergnat and I. Burghardt, Phys. Rev. Lett., 2018, 120, 227401. 
14. J. R. Mannouch, W. Barford and S. Al-Assam, J. Chem. Phys., 2018, 148, 034901.

15. J. F. Galindo, E. Atas, A. Altan, D. G. Kuroda, S. FernandezAlberti, S. Tretiak, A. E. Roitberg and V. D. Kleiman, J. Am. Chem. Soc., 2015, 137, 11637-11644.

16. F. Zheng, S. Fernandez-Alberti, S. Tretiak and Y. Zhao, J. Phys. Chem. B, 2017, 121, 5331-5339.

17. S. Athanasopoulos, L. Alfonso Hernandez, D. Beljonne, S. Fernandez-Alberti and S. Tretiak, J. Phys. Chem. Lett., 2017, 8, 1688-1694.

18. R. Franklin-Mergarejo, T. Nelson, S. Tretiak and S. FernandezAlberti, Phys. Chem. Chem. Phys., 2017, 19, 9478-9484.

19. L. Alfonso Hernandez, T. Nelson, M. F. Gelin, J. M. Lupton, S. Tretiak and S. Fernandez-Alberti, J. Phys. Chem. Lett., 2016, 7, 4936-4944.

20. R. Franklin-Mergarejo, D. Alvarez, S. Tretiak and S. FernandezAlberti, Sci. Rep., 2016, 6, 31253.

21. L. Alfonso Hernandez, T. Nelson, S. Tretiak and S. FernandezAlberti, J. Phys. Chem. B, 2015, 119, 7242-7252.

22. W. Bricker, P. Shenai, A. Ghosh, Z. Liu, M. Enriquez, P. Lambrev, H.-S. Tan, C. Lo, S. Tretiak, S. Fernandez-Alberti and Y. Zhao, Sci. Rep., 2015, 5, 13625.

23. D. Ondarse-Alvarez, N. Oldani, S. Tretiak and S. FernandezAlberti, J. Phys. Chem. A, 2014, 118, 10742-10753.

24. N. Oldani, S. Tretiak, G. Bazan and S. Fernandez-Alberti, Energy Environ. Sci., 2014, 7, 1175-1184.

25. S. Fernandez-Alberti, A. E. Roitberg, V. D. Kleiman, T. Nelson and S. Tretiak, J. Chem. Phys., 2012, 137, $22 \mathrm{~A} 526$.

26. S. Fernandez-Alberti, D. V. Makhov, S. Tretiak and D. V. Shalashilin, Phys. Chem. Chem. Phys., 2016, 18, 10028-10040. 
27. D. Ondarse-Alvarez, S. Komurlu, A. E. Roitberg, G. PierdominiciSottile, S. Tretiak, S. Fernandez-Alberti and V. D. Kleiman, Phys. Chem. Chem. Phys., 2016, 18, 25080-25089.

28. A. E. Sifain, J. A. Bjorgaard, T. R. Nelson, B. T. Nebgen, A. J. White, B. J. Gifford, D. W. Gao, O. V. Prezhdo, S. FernandezAlberti, A. E. Roitberg and S. Tretiak, J. Chem. Theory Comput., 2018, 14, 3955-3966.

29. J. Wang, J. Huang, L. Du and Z. Lan, J. Phys. Chem. A, 2015, 119, 6937-6948.

30. S. Jiang, J. Zheng, Y. Yi, Y. Xie, F. Yuan and Z. Lan, J. Phys. Chem. C, 2017, 121, 27263-27273.

31. J. Huang, L. Du, J. Wang and Z. Lan, J. Phys. Chem. C, 2015, 119, 7578-7589.

32. D. Fazzi, M. Barbatti and W. Thiel, Phys. Chem. Chem. Phys., 2015, 17, 7787-7799.

33. L. Stojanović, S. G. Aziz, R. H. Hilal, F. Plasser, T. A. Niehaus and M. Barbatti, J. Chem. Theory Comput., 2017, 13, 5846-5860.

34. J. Hoche, H.-C. Schmitt, A. Humeniuk, I. Fischer, R. Mitrić and M. I. S. Röhr, Phys. Chem. Chem. Phys., 2017, 19, 25002-25015.

35. M. I. S. Röhr, H. Marciniak, J. Hoche, M. H. Schreck, H. Ceymann, R. Mitric and C. Lambert, J. Phys. Chem. C, 2018, 122, 80828093.

36. J. C. Tully, J. Chem. Phys., 1990, 93, 1061-1071.

37. M. J. S. Dewar, E. G. Zoebisch, E. F. Healy and J. J. P. Stewart, J. Am. Chem. Soc., 1985, 107, 3902-3909.

38. S. Tretiak and S. Mukamel, Chem. Rev., 2002, 102, 3171-3212.

39. A. Humeniuk and R. Mitrić, J. Chem. Phys., 2015, 143, 134120.

40. A. Humeniuk and R. Mitrić, Comput. Phys. Commun., 2017, 221, $174-202$.

41. www.dftbaby.chemie.uni-wuerzburg.de. 
42. H. Liu, R. Wang, L. Shen, Y. Xu, M. Xiao, C. Zhang and X. Li, Org. Lett., 2017, 19, 580-583.

43. M. B. Smith and J. Michl, Chem. Rev., 2010, 110, 6891-6936.

44. J. J. Burdett and C. J. Bardeen, Acc. Chem. Res., 2013, 46, 13121320 .

45. W.-L. Chan, T. C. Berkelbach, M. R. Provorse, N. R. Monahan, J. R. Tritsch, M. S. Hybertsen, D. R. Reichman, J. Gao and X.-Y. Zhu, Acc. Chem. Res., 2013, 46, 1321-1329.

46. C. Lambert, F. Koch, S. F. Völker, A. Schmiedel, M. Holzapfel, A. Humeniuk, M. I. S. Röhr, R. Mitric and T. Brixner, J. Am. Chem. Soc., 2015, 137, 7851-7861.

47. D. J. Tozer, R. D. Amos, N. C. Handy, B. O. Roos and L. SerranoAndrés, Mol. Phys., 1999, 97, 859-868.

48. A. Dreuw, J. L. Weisman and M. Head-Gordon, J. Chem. Phys., 2003, 119, 2943-2946.

49. R. J. Magyar and S. Tretiak, J. Chem. Theory Comput., 2007, 3, 976-987.

50. Y. Kawashima, T. Hashimoto, H. Nakano and K. Hirao, Theor. Chem. Acc., 1999, 102, 49-64.

51. S. Knippenberg, J. H. Starcke, M. Wormit and A. Dreuw, Mol. Phys., 2010, 108, 2801-2813.

52. T. Zeng, R. Hoffmann and N. Ananth, J. Am. Chem. Soc., 2014, 136, 5755-5764.

53. P. M. Zimmerman, F. Bell, D. Casanova and M. Head-Gordon, J. Am. Chem. Soc., 2011, 133, 19944-19952.

54. X. Feng and A. I. Krylov, Phys. Chem. Chem. Phys., 2016, 18, $7751-7761$.

55. X. Feng, D. Casanova and A. I. Krylov, J. Phys. Chem. C, 2016, 120, 19070-19077. 
56. B. S. Basel, J. Zirzlmeier, C. Hetzer, S. R. Reddy, B. T. Phelan, M. D. Krzyaniak, M. K. Volland, P. B. Coto, R. M. Young, T. Clark, M. Thoss, R. R. Tykwinski, M. R. Wasielewski and D. M. Guldi, Chem, 2018, 4, $1092-1111$.

57. J.-D. Chai and M. Head-Gordon, Phys. Chem. Chem. Phys., 2008, 10, 6615-6620.

58. F. Weigend and R. Ahlrichs, Phys. Chem. Chem. Phys., 2005, 7, $3297-3305$.

59. A. D. Becke, J. Chem. Phys., 1993, 98, 5648-5652.

60. P. J. Stephens, F. J. Devlin, C. F. Chabalowski and M. J. Frisch, J. Phys. Chem., 1994, 98, 11623-11627.

61. T. Yanai, D. P. Tew and N. C. Handy, Chem. Phys. Lett., 2004, 393, 51-57.

62. J.-D. Chai and M. Head-Gordon, J. Chem. Phys., 2008, 128, 084106 .

63. J. Schirmer, Phys. Rev. A, 1982, 26, 2395-2416.

64. A. B. Trofimov and J. Schirmer, J. Phys. B: At., Mol. Opt. Phys., 1995, 28, 2299-2324.

65. A. Dreuw and M. Head-Gordon, Chem. Rev., 2005, 105, 40094037.

66. M. Casida and M. Huix-Rotllant, Annu. Rev. Phys. Chem., 2012, 63, 287-323.

67. A. D. McLachlan and M. A. Ball, Rev. Mod. Phys., 1964, 36, 844-855.

68. H. Nakano, R. Uchiyama and K. Hirao, J. Comp. Chem., 2002, 23, 1166-1175.

69. R. Ebisuzaki, Y. Watanabe and H. Nakano, Chem. Phys. Lett., 2007, 442, 164-169.

70. M. Miyajima, Y. Watanabe and H. Nakano, J. Chem. Phys., 2006, 124, 044101. 
71. T. H. Dunning and P. J. Hay, in Methods of Electronic Structure Theory, ed. H. F. Schaefer, Springer US, Boston, MA, 1977, pp. $1-27$.

72. M. J. Frisch, G. W. Trucks, H. B. Schlegel, G. E. Scuseria, M. A. Robb, J. R. Cheeseman, G. Scalmani, V. Barone, B. Mennucci, G. A. Petersson, H. Nakatsuji, M. Caricato, X. Li, H. P. Hratchian, A. F. Izmaylov, J. Bloino, G. Zheng, J. L. Sonnenberg, M. Hada, M. Ehara, K. Toyota, R. Fukuda, J. Hasegawa, M. Ishida, T. Nakajima, Y. Honda, O. Kitao, H. Nakai, T. Vreven, J. A. Montgomery, Jr., J. E. Peralta, F. Ogliaro, M. Bearpark, J. J. Heyd, E. Brothers, K. N. Kudin, V. N. Staroverov, R. Kobayashi, J. Normand, K. Raghavachari, A. Rendell, J. C. Burant, S. S. Iyengar, J. Tomasi, M. Cossi, N. Rega, J. M. Millam, M. Klene, J. E. Knox, J. B. Cross, V. Bakken, C. Adamo, J. Jaramillo, R. Gomperts, R. E. Stratmann, O. Yazyev, A. J. Austin, R. Cammi, C. Pomelli, J. W. Ochterski, R. L. Martin, K. Morokuma, V. G. Zakrzewski, G. A. Voth, P. Salvador, J. J. Dannenberg, S. Dapprich, A. D. Daniels, Ö. Farkas, J. B. Foresman, J. V. Ortiz, J. Cioslowski and D. J. Fox, Gaussian 09, Revision D.01, Gaussian Inc., Wallingford CT, 2009.

73. TURBOMOLE V7.0 2015, a development of University of Karlsruhe and Forschungszentrum Karlsruhe GmbH, 1989-2007, TURBOMOLE GmbH, since 200\%; available from http://www.turbomole.com.

74. W. Thiel, MNDO program, Max-Planck-Institut für Kohlensforschung, Mühlheim, Germany, $200 \%$.

75. M. W. Schmidt, K. K. Baldridge, J. A. Boatz, S. T. Elbert, M. S. Gordon, J. H. Jensen, S. Koseki, N. Matsunaga, K. A. Nguyen, S. Su et al., J. Comput. Chem., 1993, 14, 1347-1363.

76. H. J. C. Berendsen, J. P. M. Postma, W. F. van Gunsteren, A. DiNola and J. R. Haak, J. Chem. Phys., 1984, 81, 3684-3690.

77. G. Floß and P. Saalfrank, J. Phys. Chem. A, 2015, 119, 50265037.

78. G. Floß, G. Granucci and P. Saalfrank, J. Chem. Phys., 2012, 137, 234701. 
79. P. G. Lisinetskaya and R. Mitrić, Phys. Rev. A, 2011, 83, 033408.

80. G. Granucci, M. Persico and A. Toniolo, J. Chem. Phys., 2001, 114, 10608-10615.

81. F. Plasser, G. Granucci, J. Pittner, M. Barbatti, M. Persico and H. Lischka, J. Chem. Phys., 2012, 137, 22A514.

82. R. McWeeny, Rev. Mod. Phys., 1960, 32, 335-369.

83. R. Mitrić, U. Werner and V. Bonačić-Koutecký, J. Chem. Phys., 2008, 129, 164118.

84. T. Etienne, J. Chem. Phys., 2015, 142, 244103.

85. R. L. Martin, J. Chem. Phys., 2003, 118, 4775-4777.

86. M. V. Ivanov, S. A. Reid and R. Rathore, J. Phys. Chem. Lett., 2018, 9, 3978-3986. 